\title{
HYPERELLIPTIC VARIETIES
}

\author{
HERBERT LANGE
}

(Received November 24, 1999)

\begin{abstract}
A hyperelliptic variety is by definition a complex projective variety, not isomorphic to an abelian variety, which admits an abelian variety as a finite étale covering. The main contribution of this paper is a classification of hyperelliptic threefolds.
\end{abstract}

Introduction. A hyperelliptic surface (in the sense of [BPV], [GH]) is a compact complex surface, not isomorphic to an abelian surface, which admits a finite étale covering by an abelian surface. These surfaces were classified by Enriques-Severi and Bagnera-de Franchis in their fundamental papers, for which they received the Bordin prize of the French Academy of Sciences in 1907 and 1908, respectively. There are exactly five one-dimensional and two two-dimensional families of such surfaces. It seems reasonable to define more generally a hyperelliptic variety of dimension $n$ to be a complex projective variety, not isomorphic to an abelian variety, but admitting an abelian variety as a finite étale covering. It is the aim of this paper to classify hyperelliptic threefolds and to give many examples of hyperelliptic varieties in any dimensions.

The starting point of Enriques-Severi and Bagnera-de Franchis is a theorem saying that for any hyperelliptic surface $S$ there is an abelian surface $A$ admitting a finite group of biholomorphic maps $\Gamma$ acting fixed point freely on $A$, such that $S$ is isomorphic to a desingularization of $A / \Gamma$. Theorem 1.1 below implies that this result is valid for hyperelliptic varieties in any dimension $n$. Hence in order to classify hyperelliptic varieties it suffices to classify the pairs $(A, \Gamma)$ with an abelian variety $A$ and a finite group $\Gamma$ acting holomorphically and fixed point freely on $A$.

In [UY] Uchida and Yoshihara showed with a very elegant group theoretical proof that in the threefold case any such group $\Gamma$ is either cyclic of order $2,3,4,5,6,8,10,12$ or abelian of type $(2,2),(2,4),(2,6),(2,12),(3,3),(3,6),(4,4),(6,6)$ or the dihedral group $D_{4}$ of order 8 . Moreover they gave examples for these threefolds.

In this paper it is shown that the dihedral group $D_{4}$ does not occur in this list. So, if we call the finite group $\Gamma$ associated to the hyperelliptic variety, we can say that any group $\Gamma$ associated to a hyperelliptic threefold is abelian (see Theorem 6.1). For the remaining groups mentioned above we construct families of hyperelliptic varieties of dimension $n \geq 3$ associated to these groups, which in the threefold case comprise all such families. To be more precise, any biholomorphic map $f: A \rightarrow A$ of an abelian variety $A$ can be uniquely written as $f=t_{x} \circ g$ with an automorphism $g$ and a translation $t_{x}$ of $A$. The elements $g$ form a finite

2000 Mathematics Subject Classification. Primary 14J30; Secondary 14J50. 
group of automorphisms $G$. We may assume that the groups $\Gamma$ and $G$ are isomorphic. In the threefold case we compute the list of all pairs $(A, G), A$ an abelian threefold and $G$ a finite group of automorphisms for which there exists a finite group $\Gamma$ of biholomorphic maps of $A$ acting fixed point freely on $A$ and such that $\Gamma \simeq G$ under the canonical map $f=t_{x} \circ g \mapsto g$. In order to determine $\Gamma$ one has to write down only the corresponding groups of translations. This is easy for any pair $(A, G)$, but since there are many groups of such translations, the list would just be too long. So we omit writing down $\Gamma$.

The contents of the paper is as follows: In Section 1 we generalize the above mentioned theorem of Enriques-Severi and Bagnera-de Franchis to arbitrary dimensions. Section 2 gives some preliminary properties of hyperelliptic varieties. Sections 3 and 4 classify cyclic and abelian hyperelliptic varieties. In Section 5 all hyperelliptic varieties of type $(2,2)$ are constructed, and finally in Section 6 we complete classification of hyperelliptic threefolds.

Notation: If $g$ is an endomorphism of an abelian variety $A=V / \Delta, \rho_{a}(g): V \rightarrow V$ denotes the analytic representation of $g$. If $K$ denotes an algebraic subgroup of $A, K^{0}$ denotes its connected component of 0 , which is an abelian subvariety of $A$. Finally $A(n)$ denotes the group of $n$-division points of $A$ for any integer $n \geq 2$.

I am grateful to the Mathematical Institute of Tohoku University for its hospitality during the preparation of this paper.

1. The Theorem of Enriques-Severi-Bagnera-de Franchis. In [ES] Enriques and Severi and in $[\mathrm{BdF}]$ Bagnera and de Franchis proved independently the following theorem: Let $S$ be a smooth complex projective surface $S$, not rational and not an elliptic surface, admitting an abelian surface $A$ as a finite cover. Then there exists a group $\Gamma$ of biholomorphic maps of $A$ onto $A$ such that $S$ is a desingularization of $A / \Gamma$. In this section this theorem will be generalized to varieties of arbitrary dimension in the following form:

THEOREM 1.1. Let $X$ be a compact normal complex space such that there exists a complex torus $T$ and finite holomorphic map $\pi: T \rightarrow X$ of degree $d$ ramified at most in codimension $\geq 2$. Then there is a finite group $\Gamma$ of biholomorphic maps of $T$ onto $T$ such that

$$
X \simeq T / \Gamma .
$$

REMARK 1.2. Bagnera and de Franchis show that if the surface $S$ is not rational and not elliptic, the map $\pi: A \rightarrow S$ is automatically ramified at most in finitely many points. So Theorem 1.1 may be considered as a direct generalization of the Theorem of Enriques-SeveriBagnera-de Franchis.

For the proof we need some preliminaries. First of all, without loss of generality we may assume that $\pi: T \rightarrow X$ does not factorize via an isogeny $f: T \rightarrow T^{\prime}$ of complex tori. Let $T=V / \Lambda$ with a complex vector space $V$ of dimension $n$ and a lattice $\Lambda \subseteq V$. Fix a point $x_{1} \in X$ which is not a ramification point of $\pi$, let $t_{1}, \ldots, t_{d}$ be its preimages in $T$ and consider representatives $v_{1}, \ldots, v_{d}$ of $t_{i}$ in $V$. Choose open neighbourhoods $X_{1}$ of $x_{1}$ in $X$, disjoint open neighbourhoods $T_{i}$ of $t_{i}$ and $V_{i}$ of $v_{i}$ in $V$, such that 
(i) $\pi \mid T_{i} \rightarrow X_{1}$ is biholomorphic, and

(ii) $p \mid V_{i} \rightarrow T_{i}$ is biholomorphic for all $i$.

For any open set $U \subset V$ let $q_{U}$ denote the composite

$$
q_{U}:=\pi \circ p \mid U: U \rightarrow X .
$$

We mostly consider open sets $U$ such that $q_{U}$ is biholomorphic onto its image such that its inverse $q_{U}^{-1}: q_{U}(U) \rightarrow U$ is well-defined. For abbreviation we write $q_{i}=q_{V_{i}}$ and $q=q_{V}$. V.

LEMMA 1.3. The map $q_{i}^{-1} \circ q_{1}: V_{1} \rightarrow V_{i}$ extends to a biholomorphic map $\varphi_{i}: V \rightarrow$

Proof. Let $B \subset X$ be the ramification locus of $\pi$ and $A=q^{-1}(B)$, such that $q$ : $V-A \rightarrow X-B$ is étale. Let $\ell$ be a path in $V-A$ starting at $v_{1}$ and $U_{0}, \ldots, U_{t}$ any chain of overlapping open sets with the following properties:

(i) $U_{0}, \ldots, U_{t}$ are balls centered at $\ell$.

(ii) $U_{0} \subset V_{1}$ with center at $v_{1}, U_{t}$ is centered at the endpoint of $\ell$.

(iii) Let $X_{i}:=\operatorname{Im}\left(q_{i}\right)$. Then $q_{V_{i} \cup V_{i+1}}: V_{i} \cup V_{i+1} \rightarrow X_{i} \cup X_{i+1}$ is biholomorphic.

Let $W_{0}:=q_{i}^{-1}\left(X_{i}\right) \subseteq V_{i}$. Inductively one sees for $j=1, \ldots, t$ that there is a unique open set $W_{j}$ in $V$ such that

(i) $W_{j} \cap W_{j+1} \neq \emptyset$, and

(ii) $q_{W_{i}}: W_{j} \rightarrow X_{j}$ is biholomorphic for $j=1, \ldots, t$.

Then we can define the holomorphic extension $\varphi_{i}$ of $q_{i}^{-1} q_{1}: V_{1} \rightarrow V_{i}$ to $V_{1} \cup\left(\bigcup_{j=1}^{t} U_{j}\right)$ to be the composite

$$
U_{j} \stackrel{q_{U_{j}}}{\longrightarrow} X_{j} \stackrel{q_{W_{j}}^{-1}}{\longrightarrow} W_{j}
$$

on $U_{j}$. So we have the following picture:
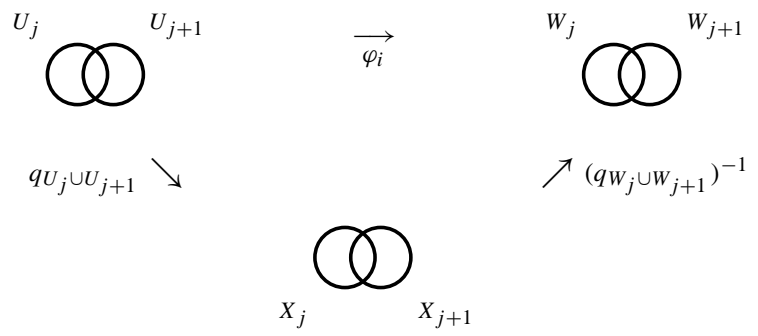

Doing this for every path in $V-A$ starting at $v_{1}$ and noting that $V-A$ is simply connected, since $A$ is of codimension $\geq 2$ in $V$, we see that $q_{i}^{-1} \circ q_{1}$ extends to a holomorphic map $\varphi_{i}$ : $V-A \rightarrow V$. By Riemann's extension theorem $\varphi_{i}$ extends holomorphically to $\varphi_{i}: V \rightarrow V$.

In the same way one shows that there is a holomorphic map $\psi_{i}: V \rightarrow V$ extending $q_{1}^{-1} \circ q_{i}: V_{i} \rightarrow V_{1}$. Since $\psi_{i} \varphi_{i}$ and $\varphi_{i} \psi_{i}$ are holomorphic extensions of the identity map on $V_{1}$ and $V_{i}$ respectively, they are both the identity map on $V$. This implies that $\varphi_{i}$ is biholomorphic. 
The map $\varphi_{i}$ depends of course on the choice of the representative $v_{i}$ of $t_{i}$. In particular, the maps $\varphi_{1}, \ldots, \varphi_{d}$ do not necessarily form a group of biholomorphic maps $V \rightarrow V$. In order to obtain a group we consider all translations by lattice points of all maps $\varphi_{i}$. Define for $i=1, \ldots, d$ and all $\lambda \in \Lambda$ the biholomorphic map

$$
\varphi_{i}^{\lambda}: V \rightarrow V \quad \text { by } \quad \varphi_{i}^{\lambda}(v)=\varphi_{i}(v)+\lambda
$$

i.e., $\varphi_{i}^{\lambda}=t_{\lambda} \circ \varphi_{i}$, where $t_{\lambda}$ denotes the translation by $\lambda$.

LEMMA 1.4. The maps $\varphi_{i}^{\lambda}, i=1, \ldots, d, \lambda \in \Lambda$, form a group $\Gamma_{0}$ of biholomorphic maps $V \rightarrow V$.

Proof. Consider the set $q^{-1}\left(x_{1}\right)=\left\{v_{i}+\lambda \mid i=1, \ldots, d, \lambda \in \Lambda\right\}$. Any biholomorphic map $\varphi_{i}^{\lambda}$ induces a permutation of the set $q^{-1}\left(x_{1}\right)$. Moreover, by construction the map $\varphi_{i}^{\lambda}$ is uniquely determined by the image of $v_{1}$ under this permutation and for every $\tilde{v} \in q^{-1}\left(x_{1}\right)$ there is exactly one biholomorphic map $\varphi_{i}^{\lambda}$ such that $\varphi_{i}^{\lambda}\left(v_{1}\right)=\tilde{v}$. Hence, if $\varphi_{i}^{\lambda_{1}}$ and $\varphi_{j}^{\lambda_{2}}$ are two such biholomorphic maps, and if $\varphi_{j}^{\lambda_{2}} \circ \varphi_{i}^{\lambda_{1}}\left(v_{1}\right)=v_{k}+\lambda_{3}$, then we have

$$
\varphi_{j}^{\lambda_{2}} \circ \varphi_{i}^{\lambda_{1}}=\varphi_{k}^{\lambda_{3}}
$$

Similarly, given $\varphi_{i}^{\lambda_{1}}$, if $v_{k}+\lambda_{0}$ is the element of $q^{-1}\left(x_{1}\right)$ with $\varphi_{i}^{\lambda_{1}}\left(v_{k}+\lambda_{0}\right)=v_{1}$, then

$$
\left(\varphi_{i}^{\lambda_{1}}\right)^{-1}=\varphi_{k}^{\lambda_{0}}
$$

This implies the assertion.

The maps $\varphi_{i}^{\lambda}$ do not necessarily descend to biholomorphic maps $T \rightarrow T$. A necessary and sufficient condition for this is that $\varphi_{i}^{\lambda}\left(v_{1}+\Lambda\right) \subseteq v_{i}+\Lambda$. But this need not be the case. However we have

LEMMA 1.5. There is a positive integer $m$ such that $\varphi_{i}^{\lambda}$ descends to a biholomorphic map

$$
{\overline{\varphi_{i}}}^{\lambda}: V / m \Lambda \rightarrow V / m \Lambda
$$

for $i=1, \ldots, d$ and all $\lambda \in \Lambda$.

PROOF. It suffices to show that for every $i=1, \ldots, d$ there is a positive integer $m_{i}$ such that

$$
\varphi_{i}\left(v_{1}+m_{i} \Lambda\right) \subseteq v_{i}+m_{i} \Lambda
$$

For every $\lambda \in \Lambda$ the map $\varphi_{i}$ induces a permutation $\sigma_{i}^{\lambda}$ of the set $\pi^{-1}\left(x_{1}\right)=\left\{t_{1}, \ldots, t_{d}\right\}$ defined by $p \varphi_{i}\left(v_{j}+\lambda\right)=t_{\sigma_{i}^{\lambda}(j)}$ for $j=1, \ldots, d$. If $\mathcal{S}_{d}$ denote the symmetric group on the set $\pi^{-1}\left(x_{1}\right)$, we obtain a permutation representation $\sigma_{i}: \Lambda \rightarrow \mathcal{S}_{d}$. Since $\operatorname{Im} \sigma_{i}$ is finite, there is a positive integer $m_{i}$ such that

$$
m_{i} \Lambda \subseteq \operatorname{ker} \sigma_{i}
$$

By construction $m_{i}$ satisfies (1.1). 
Proof of the Theorem. The map $\Gamma_{0} \rightarrow \operatorname{Bihol}(V / m \Lambda, V / m \Lambda), \varphi_{i}^{\lambda} \mapsto{\overline{\varphi_{i}}}^{\lambda}$, is a homomorphism of groups. Let $\Gamma$ denote its image. By construction $\Gamma$ is a group of biholomorphic maps on $T=V / m \Lambda$. Since moreover $X=V / \Gamma_{0}$, we obtain $X=T / \Gamma$.

2. Hyperelliptic varieties. A hyperelliptic surface is a complex projective surface, not isomorphic to an abelian surface, but admitting an abelian surface as an étale covering (see [GH], [BPV]). More generally, a hyperelliptic variety of dimension $n$ is defined to be a complex projective variety, not isomorphic to an abelian variety, but admitting an abelian variety of dimension $n$ as an étale covering. In this section some preliminaries on hyperelliptic varieties shall be given.

REMARK 2.1. (i) More generally a hyperelliptic manifold is defined to be a compact complex manifold, not isomorphic to a complex torus, but admitting a complex torus as an étale covering. There are no non-algebraic hyperelliptic surfaces. So, for dimension two both definitions coincide. However, for dimension three we will see examples of non-algebraic hyperelliptic manifolds in Remark 3.9. Since the classification of hyperelliptic threefolds given below only works in the algebraic case, we mainly stick to hyperelliptic varieties.

(ii) The notion of hyperelliptic varieties is not a generalization of the usual notion of a hyperelliptic curve. According to the genus formula of Riemann-Hurwitz there are no hyperelliptic varieties of dimension one in the above sense.

Let $X$ be a hyperelliptic variety of dimension $n$. According to Theorem 1.1 there is an abelian variety $A$ of dimension $n$ and a finite group $\Gamma$ acting holomorphically and fixed point freely on $A$ such that $X \simeq A / \Gamma$. For every $\gamma \in \Gamma$ there is a unique decomposition

$$
\gamma=t_{a} \circ g
$$

with translation $t_{a}, a \in A$ and an automorphism $g$ of $A$. This gives a map $\Gamma \rightarrow \operatorname{Aut}(A), \quad \gamma \mapsto$ $g$, which is easily seen to be a homomorphism. Let $G$ denote its image in $\operatorname{Aut}(A)$. Then there is an exact sequence

$$
0 \rightarrow T \rightarrow \Gamma \rightarrow G \rightarrow 0,
$$

where $T$ denotes a finite group of translations. Passing to the quotient abelian variety $A^{\prime}=$ $A / T$, we may assume that $T=0$, i.e., the map $\Gamma \rightarrow G, \gamma \mapsto g$, is an isomorphism. We call $G$ the group associated to the hyperelliptic variety $X$.

For any abelian variety $A$ there is a canonical exact sequence

$$
0 \rightarrow A \stackrel{i}{\rightarrow} \operatorname{Bihol}(A) \stackrel{p}{\rightarrow} \operatorname{Aut}(A) \rightarrow 0,
$$

where $\operatorname{Bihol}(A)$ denotes the group of biholomorphic maps of $A$ onto $A$, the map $i$ is defined by $a \mapsto t_{a}$ and $p$ is the canonical map derived from (2.1). Obviously (2.2) is a split exact sequence, i.e., $\operatorname{Bihol}(A)=A \rtimes \operatorname{Aut}(A)$. For any subgroup $G \subseteq \operatorname{Aut}(A)$, the sequence (2.2) induces by pullback a split exact sequence

$$
0 \rightarrow A \rightarrow \tilde{\Gamma} \stackrel{p}{\rightarrow} G \rightarrow 0 .
$$

Together with the above remarks this proves 
Proposition 2.2. For any hyperelliptic variety $X$ there is an abelian variety $A, a$ finite group of automorphisms $G$ of $A$ and a section $\sigma: G \rightarrow \tilde{\Gamma}$ of (2.3) such that the group $\Gamma=\sigma(\tilde{\Gamma})$ acts fixed point freely on $A$ and $X \simeq A / \Gamma$.

It is well-known and easy to see that the set of sections $\sigma: G \rightarrow \tilde{\Gamma}$ of (2.3) is canonically in bijection to the set of cocycles $Z^{1}(G, A)$. The following proposition gives a criterion for the cocycle to yield a fixed point free action on $A$.

Proposition 2.3. Let $\sigma: G \stackrel{\sim}{\rightarrow} \Gamma \subseteq \tilde{\Gamma}$ be a section of (2.3) with corresponding cocycle $\varphi \in Z^{1}(G, A)$. The following conditions are equivalent:

(i) $\Gamma$ acts fixed point freely on $A$.

(ii) The restriction of the cohomology class $\bar{\varphi} \in H^{1}(G, A)$ of $\varphi$ to every nontrivial cyclic subgroup of $G$ is nonzero.

Proof. $\quad \Gamma$ does not act fixed point freely if and only if there is a $g \in G, g \neq 1$ and an $a \in A$ such that $\sigma(g)(a)=a$. Since $\sigma(g)=t_{\varphi(g)} \circ g$, this means that there is a $g \in G, g \neq 1$ and an $a \in A$ such that $g(a)+\varphi(g)=a$ or equivalently $\varphi(g)=(1-g)(a)$. This means that $\varphi$ restricted to the cyclic subgroup generated by $g$ is a coboundary.

3. Cyclic hyperelliptic varieties. A hyperelliptic variety is called cyclic if the group $G$ associated to it is a cyclic group. In this section we prove a theorem classifying cyclic hyperelliptic varieties and use it to determine all such varieties in low dimensions.

So, let $X$ denote a cyclic hyperelliptic variety. We may assume that the cyclic covering $\pi: A \rightarrow X$ is minimal, that is, does not factor via an isogeny $A \rightarrow A^{\prime}$ of abelian varieties. According to Proposition 2.2 there is a biholomorphic map $f: A \rightarrow A$ of order $d=\operatorname{deg} \pi$ such that

(i) $f^{v}$ admits no fixed point and is not a translation for $1 \leq v<d$, and

(ii) $X=A /\langle f\rangle$, where $\langle f\rangle$ denotes the group generated by $f$.

There is a unique decomposition

$$
f=t_{x} \circ g
$$

with a translation $t_{x}$ and $g \in \operatorname{Aut}(A)$. This implies

$$
f^{\nu}=t_{x+g(x)+\cdots+g^{\nu-1}(x)} \circ g^{v}
$$

for all $v$. In particular, $g$ is an automorphism of order $d$ of $A$ and

$$
\sum_{\nu=0}^{d-1} g^{\nu}(x)=0 .
$$

To be more precise, $d-1$ is the smallest integer $N$ such that $\sum_{\nu=0}^{N} g^{\nu}(x)=0$, otherwise a power of $f$ would not act fixed point freely.

(3.2) immediately implies

LEMMA 3.1. The following conditions are equivalent:

(i) The group $\langle f\rangle$ acts fixed point freely on $A$.

(ii) $\sum_{i=0}^{v-1} g^{i}(x) \notin \operatorname{Im}\left(1-g^{\nu}\right)$ for all $v=1, \ldots, d-1$. 
We need the following lemma which is well-known and easy to see.

LEMMA 3.2. (a) For any endomorphism $\alpha$ of A the addition map $\mu:(\operatorname{Ker}(\alpha))^{0} \times$ $\operatorname{Im}(\alpha) \rightarrow A$ is an isogeny.

(b) $\alpha \mid \operatorname{Im}(\alpha): \operatorname{Im}(\alpha) \rightarrow \operatorname{Im}(\alpha)$ is an isogeny.

Consider

$$
B_{1}:=\operatorname{Ker}(1-g)^{0} \quad \text { and } \quad B_{2}:=\operatorname{Im}(1-g) .
$$

According to Lemma 3.2 the addition map $\mu: B_{1} \times B_{2} \rightarrow A$ is an isogeny. Moreover we have

LEMmA 3.3. (a) $B_{1}$ and $B_{2}$ are abelian subvarieties of $A$ of positive dimension.

(b) $f \mid B_{1}: B_{1} \rightarrow t_{x} B_{1}$ is a translation.

(c) $g \mid B_{1}=1_{B_{1}}$.

(d) $g \mid B_{2}$ is an automorphism of $B_{2}$ with finitely many fixed points.

(e) $B_{1} \cap B_{2} \subseteq \operatorname{Fix}\left(g \mid B_{2}\right)$ which is a finite set.

(f) $(1-g) \mid B_{2}$ is an isogeny of $B_{2}$.

Proof. (a) If $1-g$ is an isogeny, then $f$ always admits fixed points according to Lemma 3.1. Hence $\operatorname{dim} B_{1}>0$. On the other hand, $1-g \neq 0$, otherwise $f$ would be a translation. Hence $\operatorname{dim} B_{2}>0$. (b) and (c) are obvious.

(d) and (e). Suppose $x \in B_{2}$. There is a $y \in A$ with $x=y-g(y)$ implying $g(x)=$ $(1-g)(g(y)) \in \operatorname{Im}(1-g)=B_{2}$. So $g \mid B_{2}$ is an automorphism of $B_{2}$, since $g$ is injective as an automorphism of $A$. The fixed points of $g \mid B_{2}$ are just the points of the intersection $\operatorname{Ker}(1-g) \cap B_{2}$, which is finite. (f) is a consequence of Lemma $3.2(b)$.

Choose a decomposition $x=x_{1}+x_{2}$ with $x_{1} \in B_{1}$ and $x_{2} \in B_{2}$, and define

$$
\tilde{f}:=t_{\left(x_{1}, x_{2}\right)} \circ(1 \times g) .
$$

Then the following diagram is commutative

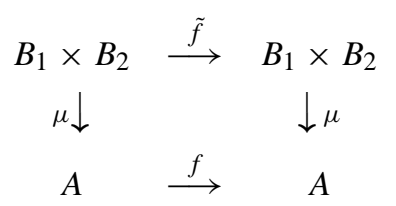

LEMMA 3.4. Let $T$ denote the group of translations $t_{(y,-y)}$ of $B_{1} \times B_{2}$ with $y \in B_{1} \cap B_{2}$ and $G:=\langle\tilde{f}\rangle \oplus T$. Then

$$
X \simeq B_{1} \times B_{2} / G .
$$

Proof. It suffices to show that $t_{(y,-y)}$ and $\tilde{f}$ commute for any $y \in B_{1} \cap B_{2}$, which is an immediate computation using $y \in \operatorname{Fix}\left(g \mid B_{2}\right)$.

The decomposition (3.4) depends of course on the choice of the group structure, i.e., 
on the choice of the zero point of $B_{1} \times B_{2}$. The following lemma uses this fact in order to normalize the decomposition.

LEMMA 3.5. Choosing a suitable zero point of $B_{1} \times B_{2}$, we may assume that

$$
\tilde{f}=t_{\left(x_{1}, 0\right)} \circ(1 \times g)
$$

with $x_{1} \in B_{1}$ as above and $g \in \operatorname{Aut}\left(B_{2}\right)$.

ProOF. Let $\tilde{f}=t_{\left(x_{1}, x_{2}\right)} \circ(1 \times g)=\left(f_{i j}\right)_{i, j=1,2}: B_{1} \times B_{2} \rightarrow B_{1} \times B_{2}$ be as in (3.4). Then $f_{22}: B_{2} \rightarrow B_{2}$ is given by $f_{22}(z)=g(z)+x_{2}$. Let $y_{0} \in B_{2}$ with $(1-g)\left(y_{0}\right)=x_{2}$. Then

$$
f_{22}\left(y_{0}\right)=g\left(y_{0}\right)+x_{2}=y_{0} .
$$

Choose $\left(0, y_{0}\right)$ as the new zero point of $B_{1} \times B_{2}$ and let $\tilde{f}=t_{\left(x_{1}^{\prime}, x_{2}^{\prime}\right)} \circ(1 \times \tilde{g})$ be the decomposition of $\tilde{f}$ with respect to $\left(0, y_{0}\right)$. We have to compute $\left(x_{1}^{\prime}, x_{2}^{\prime}\right)$. But $\tilde{g}=t_{y_{0}} \circ g \circ t_{-y_{0}}$ and hence

$$
\begin{aligned}
t_{\left(x_{1}, 0\right)}(1 \times \tilde{g})\left(z_{1}, z_{2}\right) & =\left(z_{1}+x_{1}, g\left(z_{2}\right)-g\left(y_{0}\right)+y_{0}\right) \\
& =\left(z_{1}+x_{1}, g\left(z_{2}\right)+x_{2}\right) \\
& =\tilde{f}\left(z_{1}, z_{2}\right) .
\end{aligned}
$$

The elements of $G=\langle\tilde{f}\rangle \oplus T$ are of the form $t_{\left(x_{1}, x_{2}\right)} \circ\left(1 \times g^{i}\right)$ with $0 \leq i<d$ and $x_{1} \in B_{1}, x_{2} \in B_{2}$ torsion points. To be more precise, $x_{2}=0$ if $i>0$ and $x_{2}=-x_{1} \in B_{1} \cap B_{2}$ if $i=0$. In particular, we have a well-defined map

$$
\varphi: G \rightarrow B_{1}, \quad t_{\left(x_{1}, x_{2}\right)} \circ\left(1 \times g^{i}\right) \mapsto x_{1} .
$$

LEMMA 3.6. $\varphi: G \rightarrow B_{1}$ is an injective homomorphism of groups.

Proof. $\quad t_{\left(x_{1}, x_{2}\right)}\left(1 \times g^{j}\right) \circ t_{\left(y_{1}, y_{2}\right)}\left(1 \times g^{i}\right)=t_{\left(x_{1}+y_{1}, x_{2}+g^{i}\left(y_{2}\right)\right)}\left(1 \times g^{i+j}\right)$, and hence $\varphi$ is a homomorphism of groups. Any element of $\operatorname{Ker}(\varphi)$ is of the form $t_{\left(0, x_{2}\right)}\left(1 \times g^{i}\right)$. If $0<i<d$, then this element has fixed points, and hence is not contained in $G$.

COROLlaRY 3.7. The group of translations $T$ is a finite abelian group with $\leq$ $2 \operatorname{dim} B_{1}-1$ generators.

This follows from the fact that any finite torsion subgroups of $B_{1}$ is generated by $\leq$ $2 \operatorname{dim} B_{1}$ elements.

According to Lemma 3.6 we may consider $G$ as a subgroup of $B_{1}$. Moreover the map

$$
\rho: G \rightarrow \operatorname{Bihol}\left(B_{2}\right), \quad x_{1} \mapsto t_{x_{2}} g^{i},
$$

if $\varphi^{-1}\left(x_{1}\right)=t_{\left(x_{1}, x_{2}\right)}\left(1 \times g^{i}\right)$, is a faithful representation. Combining everything we have:

THEOREM 3.8. For a variety $X$ of dimension $n$ the following statements are equivalent:

(i) $X$ is a cyclic hyperelliptic variety. 
(ii) There are abelian varieties $B_{1}$ of dimension $0<n_{1}<n, B_{2}$ of dimension $n_{2}=$ $n-n_{1}$, a finite subgroup $G=\left\langle x_{1}\right\rangle \oplus T$ of $B_{1}$, and a faithful representation $\rho: G \rightarrow \operatorname{Bihol}\left(B_{2}\right)$ such that

(a) $g=\rho\left(x_{1}\right)$ is an automorphism of $B_{2}$ of order $d \geq 2$ with $\operatorname{Fix}(g)$ finite, and

(b) $\quad \rho(T)$ is a group of translations of $B_{2}$ by elements of $\operatorname{Fix}(g)$.

If an action of $G$ on $B_{1} \times B_{2}$ is defined by $\left(x,\left(z_{1}, z_{2}\right)\right) \rightarrow\left(t_{x}\left(z_{1}\right), \rho(x) z_{2}\right)$, then $X \simeq$ $B_{1} \times B_{2} / G$.

Proof. It remains to show that under condition (ii) the group $G$ acts fixed point freely on $B_{1} \times B_{2}$. Let $\tilde{f}: B_{1} \times B_{2} \rightarrow B_{1} \times B_{2}$ be defined by $\tilde{f}=t_{\left(x_{1}, 0\right)} \circ(1 \times g)$, and $\tilde{T}=\left\{(x, \rho(x)(0)) \in B_{1} \times B_{2} \mid x \in T\right\}$ considered as a group of translations of $B_{1} \times B_{2}$. Then any element of $\langle\tilde{f}\rangle \oplus \tilde{T} \simeq G$ is of the form

$$
\tilde{f}^{i} \circ t_{(x, \rho(x)(0))}
$$

with $0 \leq i \leq d-1$ and $x \in T$, and we have

$$
\tilde{f}^{i} \circ t_{(x, \rho(x)(0))}\left(z_{1}, z_{2}\right)=\left(z_{1}+x+i x_{1}, g^{i}\left(z_{2}\right)+\rho(x)(0)\right) .
$$

To see this one has to use that $\rho(x)(0) \subseteq \operatorname{Fix}(g)$. Now suppose $\left(b_{1}, b_{2}\right) \in B_{1} \times B_{2}$ is a fixed point of $\tilde{f}^{i} \circ t_{(x, \rho(x)(0))}$. This implies $b_{1}+x+i x_{1}=b_{1}$ and thus $x=-i x_{1} \neq 0$ contradicting the fact that $\left\langle x_{1}\right\rangle \oplus T$ is a direct sum in $B_{1}$.

Since all automorphisms of finite order of abelian varieties of dimension $\leq 3$ are wellknown, one can use Theorem 3.8 to give a list of all cyclic hyperelliptic varieties with $\operatorname{dim}\left(B_{2}\right)=r \leq 3$. For this one has only to give a list of all automorphisms of finite order of abelian varieties of dimension $r$ together with their fixed point sets Fix $(g)$. In order to define a hyperelliptic variety one has to give only an abelian variety $B_{1}$ an element $x_{1} \in B_{1}$ of order $d=\operatorname{ord}(g)$, a finite subgroup $T \subset B_{1}$ not intersecting the group $\left\langle x_{1}\right\rangle$ and an embedding $T \hookrightarrow \operatorname{Fix}(g)$. Tables 1 and 2 below give the triples $\left(B_{2}, g\right.$, Fix $\left.(g)\right)$ for all cyclic hyperelliptic varieties $\operatorname{dim}\left(B_{2}\right)=1$ and 2 . From this it is easy to work out the other data. The notation will be explained after the tables.

TABLE $1 . \quad \operatorname{dim}\left(B_{2}\right)=1$.

\begin{tabular}{|c|c|c|c|}
\hline$d$ & $B_{2}$ & $g$ & $\operatorname{Fix}(g)$ \\
\hline \hline 2 & $E$ & -1 & $E(2)$ \\
\hline 3 & $E_{\rho}$ & $\rho$ & $\left\{\frac{v}{3}(2+\rho) \mid v=0,1,2\right\}$ \\
\hline 4 & $E_{i}$ & $i$ & $\left\{\frac{v}{2}(1+i) \mid v=0,1\right\}$ \\
\hline 6 & $E_{\rho}$ & $-\rho$ & $\{0\}$ \\
\hline
\end{tabular}


TABLE 2. $\operatorname{dim}\left(B_{2}\right)=2$.

\begin{tabular}{|c|c|c|c|}
\hline$d$ & $B_{2}$ & $g$ & $\operatorname{Fix}(g)$ \\
\hline 2 & $S$ & -1 & $S(2)$ \\
\hline 3 & $C^{2} / \Pi_{1} Z^{4}$ & $\left(\begin{array}{ll}0 & -1 \\
1 & -1\end{array}\right)$ & $\left\{\left(\begin{array}{c}x \\
-x\end{array}\right) \mid 3\left(\begin{array}{c}x \\
-x\end{array}\right) \in \Pi_{1} z\right\}$ \\
\hline 3 & $E_{\rho} \times E_{\rho}$ & $\left(\begin{array}{cc}\rho & 0 \\
0 & \rho^{j}\end{array}\right) j=1,2$ & $\operatorname{Fix}(\rho) \times \operatorname{Fix}(\rho)$ \\
\hline 4 & $C^{2} / \Pi_{2} Z^{4}$ & $\left(\begin{array}{cc}0 & -1 \\
1 & 0\end{array}\right)$ & $\left\{\left(\begin{array}{c}x \\
-x\end{array}\right) \mid 2\left(\begin{array}{c}x \\
-x\end{array}\right) \in \Pi_{2} \boldsymbol{Z}\right\}$ \\
\hline 4 & $E_{i} \times E_{i}$ & $\left(\begin{array}{cc}i & 0 \\
0 & j\end{array}\right) j=1,3$ & $\operatorname{Fix}(i) \times \operatorname{Fix}(i)$ \\
\hline 4 & $E \times E_{i}$ & $\left(\begin{array}{cc}-1 & 0 \\
0 & i\end{array}\right)$ & $E(2) \times \operatorname{Fix}(i)$ \\
\hline 5 & $S_{5}$ & $\left(\begin{array}{cc}\xi_{5} & 0 \\
0 & \xi_{5}^{3}\end{array}\right)$ & $\left\{\frac{1}{5}\left(1+2 \xi_{5}^{v}+3 \xi_{5}^{2 v}+4 \xi_{5}^{3 v}\right) \mid v=0, \ldots, 4\right\}$ \\
\hline 6 & $C^{2} / \Pi_{1} Z^{4}$ & $\left(\begin{array}{cc}0 & 1 \\
-1 & 1\end{array}\right)$ & $\{0\}$ \\
\hline 6 & $E_{\rho} \times E_{\rho}$ & $\left(\begin{array}{cc}-\rho & 0 \\
0 & -\rho^{j}\end{array}\right) j=1,2$ & $\{0\}$ \\
\hline 6 & $E_{\rho} \times E_{\rho}$ & $\left(\begin{array}{cc}\rho & 0 \\
0 & -\rho\end{array}\right)$ & $\operatorname{Fix}(\rho) \times\{0\}$ \\
\hline 6 & $E_{\rho} \times E_{\rho}$ & $\left(\begin{array}{cc}\rho & 0 \\
\pm \rho & -\rho\end{array}\right)$ & $\{0\}$ \\
\hline 6 & $E_{\rho} \times E$ & $\left(\begin{array}{cc}-\rho & 0 \\
0 & -1\end{array}\right)$ & $\{0\} \times E(2)$ \\
\hline 6 & $E_{\rho} \times E$ & $\left(\begin{array}{cc}\rho & 0 \\
0 & -1\end{array}\right)$ & $\operatorname{Fix}(\rho) \times E(2)$ \\
\hline 8 & $E_{i} \times E_{i}$ & $\left(\begin{array}{ll}0 & i \\
1 & 0\end{array}\right)$ & $\{(x, x) \mid x \in \operatorname{Fix}(i)\}$ \\
\hline 8 & $E_{\sqrt{-2}} \times E_{\sqrt{-2}}$ & $\left(\begin{array}{cc}\sqrt{-2} & -1 \\
-1 & 0\end{array}\right)$ & $\left\{\frac{i}{2}, 0\right\}$ \\
\hline 10 & $S_{5}$ & $\left(\begin{array}{cc}\xi_{10} & 0 \\
0 & \xi_{10}^{3}\end{array}\right)$ & $\{0\}$ \\
\hline 12 & $E_{i} \times E_{i}$ & $\left(\begin{array}{cc}0 & i \\
-i & -i\end{array}\right)$ & $\{0\}$ \\
\hline 12 & $E_{\rho} \times E_{\rho}$ & $\left(\begin{array}{cc}0 & \rho \\
-\rho & 0\end{array}\right)$ & $\{0\}$ \\
\hline 12 & $E_{i} \times E_{\rho}$ & $\left(\begin{array}{ll}i & 0 \\
0 & \rho\end{array}\right)$ & $\operatorname{Fix}(i) \times \operatorname{Fix}(\rho)$ \\
\hline 12 & $E_{i} \times E_{\rho}$ & $\left(\begin{array}{cc}i & 0 \\
0 & -\rho\end{array}\right)$ & $\operatorname{Fix}(i) \times\{0\}$ \\
\hline
\end{tabular}

Here $E$ (resp. $S$ ) is an arbitrary elliptic curve (resp. abelian surface), $E_{\tau}=C /(1, \tau) Z$ for any $\tau$ in the upper half plane, $\xi_{d}$ denotes a primitive $d$-th root of unity, and we abbreviate $i=\xi_{4}, \rho=\xi_{3}$. Moreover, $S_{5}$ denotes the abelian surface of $C M$-type $\left(\boldsymbol{Q}\left(\xi_{5}\right),\left(\xi_{5}, \xi_{5}^{2}\right)\right)$, and 
$\Pi_{1}$ and $\Pi_{2}$ the period matrices

$$
\Pi_{1}=\left(\begin{array}{cccc}
1 & 0 & x & y \\
0 & 1 & -y & x+y
\end{array}\right) ; \Pi_{2}=\left(\begin{array}{cccc}
1 & 0 & x & y \\
0 & 1 & -y & x
\end{array}\right)
$$

with $(x, y) \in \boldsymbol{C}^{2}-\boldsymbol{R}^{2}$, defining abelian varieties.

Table 2 uses heavily Fujiki's list of automorphisms of abelian surfaces [F].

REMARK 3.9. (a) The quotient $\boldsymbol{C}^{2} / \Pi_{i} \boldsymbol{Z}^{4}$ is a complex torus for every $(x, y) \in \boldsymbol{C}^{2}-$ $\boldsymbol{R}^{2}$, but not always an abelian surface. In the cases where it is not, the quotient $X=B_{1} \times B_{2} / \Gamma$ is a non algebraic hyperelliptic surface. Since the existence of non algebraic hyperelliptic varieties contradicts Theorem II of [J], we give an explicit example: The complex torus $B_{2}=$ $C^{2} / \Pi Z^{4}$ with

$$
\Pi=\left(\begin{array}{cccc}
1 & i & 0 & \alpha \\
0 & 0 & 1 & i
\end{array}\right)
$$

fits into an exact sequence $0 \rightarrow E_{i} \rightarrow B_{2} \rightarrow E_{i} \rightarrow 0$. If $\alpha \notin Q(i)$, then $E_{i}$ is the only nontrivial complex subtorus of $S$ (see [BL] Section 1.6). In particular, $B_{2}$ is a non algebraic complex torus. Its automorphism group is isomorphic to $Z / 4 Z$ and generated by $g=\left(\begin{array}{cc}i & -\alpha \\ 0 & -i\end{array}\right)$ and Fix $(g)=\{0, \overline{(x, y)}\}$ with $x=-(i / 2) \alpha, y=(1-i) / 2$. Choosing an elliptic curve $B_{1}$ and a 4-division point $x_{1} \in B_{1}$, the group $G$ acts on $B_{1} \times B_{2}$ by $t_{x_{1}} \circ(1 \times g)$ and its quotient is a non algebraic hyperelliptic threefold.

(b) The automorphism of 3-dimensional abelian varieties were classified in [BGL]. Therefore one could also write a table of cyclic hyperelliptic varieties of order 3 . This will be omitted, since the table would be too long.

4. Abelian hyperelliptic varieties. A hyperelliptic variety is called abelian if its associated group $G$ is an abelian group. In this section we prove a theorem describing such varieties for abelian groups with two generators, which allows to construct abelian hyperelliptic varieties in any dimensions and will be applied in Section 6 to give a list of all abelian hyperelliptic threefolds.

Let $A$ be an abelian variety of dimension $n(\geq 3)$ and $G$ a group of automorphisms of $A$ isomorphic to $\boldsymbol{Z} / d_{1} \boldsymbol{Z} \oplus \boldsymbol{Z} / d_{2} \boldsymbol{Z}$ with $d_{1} \mid d_{2}$. Suppose $\Gamma$ is a group of biholomorphic maps of $A$, isomorphic to $G$ and acting fixed point freely on $A$. Then

$$
X:=A / \Gamma
$$

is an abelian hyperelliptic variety associated to the group $G$. We call it of type $\left(d_{1}, d_{2}\right)$. Let $g_{1}$ and $g_{2}$ be automorphisms of $A$ of order $d_{1}$ and $d_{2}$ generating $G$, and $f_{1}, f_{2}$ the corresponding generators of $\Gamma$

$$
f_{1}=t_{x} \circ g_{1} \text { and } f_{2}=t_{x^{\prime}} \circ g_{2} .
$$

Define abelian subvarieties $A_{i}$ by

$$
\begin{array}{ll}
A_{1}=\left(\operatorname{Ker}\left(1-g_{1}\right) \cap \operatorname{Ker}\left(1-g_{2}\right)\right)^{0}, & A_{2}=\left(\operatorname{Ker}\left(1-g_{1}\right) \cap \operatorname{Im}\left(1-g_{2}\right)\right)^{0}, \\
A_{3}=\left(\operatorname{Im}\left(1-g_{1}\right) \cap \operatorname{Ker}\left(1-g_{2}\right)\right)^{0}, & A_{4}=\left(\operatorname{Im}\left(1-g_{1}\right) \cap \operatorname{Im}\left(1-g_{2}\right)\right)^{0} .
\end{array}
$$


Applying Lemma 3.2 twice, we conclude that the addition map induces an isogeny $A_{1} \times A_{2} \times$ $A_{3} \times A_{4} \rightarrow A$. In this section we assume that $B_{1}:=A_{1}$ is positive dimensional. If $B_{2}$ denotes the image of the induced map $A_{2} \times A_{3} \times A_{4} \rightarrow A$, we obtain that the addition map induces an isogeny

$$
\mu: B_{1} \times B_{2} \rightarrow A .
$$

The maps $g_{1}$ and $g_{2}$ restrict to the identity on $B_{1}$ and to automorphisms of $B_{2}$, which we also denote by $g_{1}$ and $g_{2}$.

LEMMA 4.1. $\quad B_{1} \cap B_{2} \subset \operatorname{Fix}\left(g_{1} \mid B_{2}\right) \cap \operatorname{Fix}\left(g_{2} \mid B_{2}\right)$ which is finite.

Proof. The only nontrivial assertion is that $\operatorname{Fix}\left(g_{1} \mid B_{2}\right) \cap \operatorname{Fix}\left(g_{2} \mid B_{2}\right)$ is a finite set. But

$$
\operatorname{Fix}\left(g_{1} \mid B_{2}\right) \subset\left(\operatorname{Ker}\left(1-g_{1}\right) \cap \operatorname{Im}\left(1-g_{1}\right)\right) \cup\left(\operatorname{Ker}\left(1-g_{1}\right) \cap \operatorname{Im}\left(1-g_{2}\right)\right) .
$$

The first set is finite by Lemma 3.2(a) and the second is a union of finitely many translates of $A_{2}$. Similarly,

$$
\operatorname{Fix}\left(g_{2} \mid B_{2}\right) \subset\left(\operatorname{Ker}\left(1-g_{2}\right) \cap \operatorname{Im}\left(1-g_{2}\right)\right) \cup\left(\operatorname{Ker}\left(1-g_{2}\right) \cap \operatorname{Im}\left(1-g_{1}\right)\right) .
$$

Again the first set is finite by Lemma 3.2(a) and the second is a union of finitely many translates of $A_{3}$. Hence the assertion follows from the fact that $A_{2} \cap A_{3}$ is finite.

Consider decompositions

$$
x=x_{1}+x_{2}, \quad x^{\prime}=x_{1}^{\prime}+x_{2}^{\prime}
$$

with $x_{1}, x_{1}^{\prime} \in B_{1}$ and $x_{2}, x_{2}^{\prime} \in B_{2}$.

LEMMA 4.2. (a) $d_{1} x_{1}=-\sum_{\nu=0}^{d_{1}-1} g_{1}^{\nu}\left(x_{2}\right) \in B_{1} \cap B_{2}$.

(b) $d_{2} x_{1}^{\prime}=-\sum_{v=0}^{d_{2}-1} g_{2}^{\nu}\left(x_{2}^{\prime}\right) \in B_{1} \cap B_{2}$.

Proof. According to Equation (3.3), $\sum_{v=0}^{d_{1}-1} g_{1}^{v}(x)=0$. This implies (a), since $g_{1}\left(x_{1}\right)=x_{1}$. The proof of $(\mathrm{b})$ is the same.

Define biholomorphic maps $\tilde{f}_{1}$ and $\tilde{f}_{2}: B_{1} \times B_{2} \rightarrow B_{1} \times B_{2}$ by

$$
\tilde{f}_{1}:=t_{\left(x_{1}, x_{2}\right)} \circ\left(1 \times g_{1}\right) \quad \text { and } \quad \tilde{f}_{2}:=t_{\left(x_{1}^{\prime}, x_{2}^{\prime}\right)} \circ\left(1 \times g_{2}\right) .
$$

For $i=1$ and 2 the following diagram is commutative

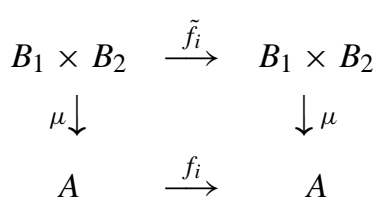

LEMMA 4.3 . (a) $\tilde{f}_{1}^{d_{1}}=t_{\left(d_{1} x_{1}, \sum_{v=0}^{d_{1}-1} g_{1}^{v}\left(x_{2}\right)\right)}, \quad \tilde{f}_{2}^{d_{2}}=t_{\left(d_{2} x_{1}^{\prime}, \sum_{v=0}^{d_{2}-1} g_{2}^{v}\left(x_{2}^{\prime}\right)\right)}$.

(b) $\left(1-g_{1}\right)\left(x_{2}^{\prime}\right)=\left(1-g_{2}\right)\left(x_{2}\right)$. 
ProOF. (a) is a consequence of $f_{i}^{d_{i}}=1$ for $i=1$, 2. For (b) note that $f_{1} f_{2}=f_{2} f_{1}$ implies $g_{1}\left(x^{\prime}\right)+x=g_{2}(x)+x^{\prime}$. This gives the assertion since $g_{1}\left(x_{1}^{\prime}\right)=x_{1}^{\prime}$ and $g_{2}\left(x_{1}\right)=$ $x_{1}$.

LEMMA 4.4. Let $T$ denote the group of translations $t_{(b,-b)}$ of $B_{1} \times B_{2}$ with $b \in B_{1} \cap$ $B_{2}$. Then the subgroup $\tilde{\Gamma}$ of $\operatorname{Bihol}\left(B_{1} \times B_{2}\right)$ generated by $\tilde{f}_{1}, \tilde{f}_{2}$ and $T$ is a finite abelian group with

$$
X \simeq B_{1} \times B_{2} / \tilde{\Gamma}
$$

Proof. It suffices to show that $\tilde{f}_{1}, \tilde{f}_{2}$ and $T$ commute. But this is an immediate computation using Lemma 4.3 and $g_{1}(b)=g_{2}(b)=b$.

Combining everything we have proved part of the following theorem.

THEOREM 4.5. For a variety $X$ of dimension $n(\geq 3)$ and positive integers $d_{1}, d_{2}$ with $d_{1} \mid d_{2}$ the following statements are equivalent:

(i) $X$ is an abelian hyperelliptic variety of type $\left(d_{1}, d_{2}\right)$.

(ii) There are

- abelian varieties $B_{1}$ and $B_{2}$ with $\operatorname{dim} B_{1}+\operatorname{dim} B_{2}=n$,

- commuting automorphisms $g_{i}$ of $B_{2}$ of order $d_{i}$ for $i=1,2$ with $\operatorname{Fix}\left(g_{1}\right) \cap \operatorname{Fix}\left(g_{2}\right)$ finite,

- points $\left(x_{1}, x_{2}\right)$ and $\left(x_{1}^{\prime}, x_{2}^{\prime}\right) \in B_{1} \times B_{2}$ with $\left(1-g_{1}\right)\left(x_{2}^{\prime}\right)=\left(1-g_{2}\right)\left(x_{2}\right)$,

- a subgroup $\tau$ of $\operatorname{Fix}\left(g_{1}\right) \cap \operatorname{Fix}\left(g_{2}\right)$ containing $\sum_{v=0}^{d_{1}-1} g_{1}^{v}\left(x_{2}\right)$ and $\sum_{v=0}^{d_{2}-1} g_{2}^{v}\left(x_{2}^{\prime}\right)$ and an injective homomorphism $\iota: \tau \rightarrow B_{1}$ with $\iota\left(\sum_{v=0}^{d_{1}-1} g_{1}^{\nu}\left(x_{2}\right)\right)=-d_{1} x_{1}$ and such that $\iota\left(\sum_{v=0}^{d_{2}-1} g_{2}^{\nu}\left(x_{2}^{\prime}\right)\right)=-d_{2} x_{1}^{\prime}$,

(a) $\quad X \simeq B_{1} \times B_{2} / \Gamma$, where $\Gamma$ is the subgroup of $\operatorname{Bihol}\left(B_{1} \times B_{2}\right)$ generated by $f_{1}=$ $t_{\left(x_{1}, x_{2}\right)} \circ\left(1 \times g_{1}\right), f_{2}=t_{\left(x_{1}^{\prime}, x_{2}^{\prime}\right)} \circ\left(1 \times g_{2}\right)$ and $T=\left\{t_{(\iota(y), y)} \mid y \in \tau\right\}$, and

(b) for all $i=1, \ldots, d_{1}-1, j=1, \ldots, d_{2}-1$ and $y \in \tau$ we have

$$
\begin{aligned}
& j x_{1}^{\prime}+i x_{1}+\iota(y) \neq 0 \quad \text { or } \\
& \sum_{\nu=0}^{i-1} g_{1}^{\nu}\left(x_{2}\right)+\sum_{\nu=0}^{j-1} g_{1}^{i} g_{2}^{\nu}\left(x_{2}^{\prime}\right) \notin \operatorname{Im}\left(g_{1}^{i} g_{2}^{j}\right) .
\end{aligned}
$$

Proof. Note first that $\Gamma$ is a finite abelian subgroup of $\operatorname{Bihol}\left(B_{1} \times B_{2}\right)$. This follows from the assumptions with the same computations as in the proof of Lemma 4.4. It remains to show that $\Gamma$ acts fixed point freely on $B_{1} \times B_{2}$ if and only if Condition (b) is satisfied. But this follows immediately from the fact that the elements of $\Gamma$ are just

$$
t_{(\iota(y), y)} \circ f_{1}^{i} \circ f_{2}^{j}=t_{\left.\left(i x_{1}+j x_{1}^{\prime}+\iota(y)\right), \sum_{v=0}^{i-1} g_{1}^{v}\left(x_{2}\right)+\sum_{v=0}^{j-1} g_{1}^{i} g_{2}^{v}\left(x_{2}^{\prime}\right)+y\right)} \circ\left(1 \times g_{1}^{i} g_{2}^{j}\right)
$$

with $1 \leq i \leq d_{1}-1,1 \leq j \leq d_{2}-1$ and $y \in \tau$. 
The easiest way to construct abelian hyperelliptic varieties is given by the following corollary.

COROLlaRY 4.6. Suppose we are given abelian varieties $B_{1}$ of dimension $n_{1}>0$ and $B_{2}$ of dimension $n-n_{1}>0$, a finite subgroup $\Gamma=\left\langle x_{1}\right\rangle \oplus\left\langle x_{1}^{\prime}\right\rangle \oplus T$ of $B_{1}$ and a faithful representation $\rho: \Gamma \rightarrow \operatorname{Bihol}\left(B_{2}\right)$ such that

(a) $\quad \rho\left(\left\langle x_{1}\right\rangle \oplus\left\langle x_{1}^{\prime}\right\rangle\right)$ is a group of automorphisms of $\boldsymbol{B}_{2}$ isomorphic to $\boldsymbol{Z} / d_{1} \boldsymbol{Z} \oplus \boldsymbol{Z} / d_{2} \boldsymbol{Z}$ with $\operatorname{Fix}\left(\rho\left(x_{1}\right)\right) \cap \operatorname{Fix}\left(\rho\left(x_{1}^{\prime}\right)\right)$ finite.

(b) $\quad \rho(T)$ is a group of translations of $B_{2}$ by elements of $\operatorname{Fix}\left(\rho\left(x_{1}\right)\right) \cap \operatorname{Fix}\left(\rho\left(x_{1}^{\prime}\right)\right)$. If $G$ acts on $B_{1} \times B_{2}$ by $\left(x,\left(b_{1}, b_{2}\right)\right) \mapsto\left(t_{x}\left(b_{1}\right), \rho(x) b_{2}\right)$, then

$$
X \simeq B_{1} \times B_{2} / \Gamma
$$

is an abelian hyperelliptic variety of dimension $n$ of type $\left(d_{1}, d_{2}\right)$.

Proof. Choose $x_{2}=x_{2}^{\prime}=0$. Let $\tau$ denote the subgroup $\tau=\{\rho(t)(0) \mid t \in T\}$ of $B_{2}$ and $\iota: \tau \rightarrow T$ the obvious isomorphism. Then all the conditions of (ii) of Theorem 4.5 are satisfied.

REMARK 4.7. (a) One can also easily prove Corollary 4.6 directly without using Theorem 4.5. Moreover, it seems obvious how to generalize the corollary to construct abelian hyperelliptic varieties of arbitrary type $\left(d_{1}, \ldots, d_{v}\right)$. We will omit this, since it will not be used in the sequel.

(b) Theorem 4.5 is trivially valid also for $\operatorname{dim} B_{1}=0$. In fact, in this case Condition (ii) reduces to the definition of a hyperelliptic variety associated to the group $G$.

(c) One might try to obtain a better description of abelian hyperelliptic varieties of type $\left(d_{1}, d_{2}\right)$ using the isogeny $\mu: A_{1} \times A_{2} \times A_{3} \times A_{4} \rightarrow A$ of the beginning of this section. In fact, $f_{1}$ and $f_{2}$ lift to biholomorphic maps of $A_{1} \times \cdots \times A_{4}$, and so this can be done. However, there are some difficulties to the effect that the result seems not easier to apply than Theorem 4.5: First of all, the kernel of $\mu$ seems complicated. Moreover, the liftings of $f_{1}$ and $f_{2}$ do not commute in general. These difficulties vanish in the special case $\left(d_{1}, d_{2}\right)=(2,2)$ as we shall see in the next section.

5. Abelian hyperelliptic varieties of type (2,2). For a hyperelliptic variety $X$ associated to a group $G \simeq \boldsymbol{Z} / 2 \boldsymbol{Z} \times \boldsymbol{Z} / 2 \boldsymbol{Z}$ one can use the isogeny $A_{1} \times A_{2} \times A_{3} \times A_{4} \rightarrow X$ of the last section to obtain a better description of $X$.

Let the notation be as at the beginning of the last section with $d_{1}=d_{2}=2$. So, $\Gamma$ is a group of biholomorphic maps, isomorphic to $\boldsymbol{Z} / 2 \boldsymbol{Z} \oplus \boldsymbol{Z} / 2 \boldsymbol{Z}$, generated by $f_{1}=t_{x} \circ g_{1}$ and $f_{2}=t_{x^{\prime}} \circ g_{2}$ and acting fixed point freely on an abelian variety $A$ of dimension $n(\geq 3)$ such that $X \simeq A / \Gamma$. Moreover

$$
\mu: A_{1} \times A_{2} \times A_{3} \times A_{4} \rightarrow A
$$

is an isogeny, where $A_{1}, \ldots, A_{4}$ are abelian subvarieties of $A$ defined as above. Here we have

$$
\begin{array}{ll}
g_{1} \mid A_{1} \times A_{2}=1, & g_{1} \mid A_{3} \times A_{4}=-1, \\
g_{2} \mid A_{1} \times A_{3}=1, & g_{2} \mid A_{2} \times A_{4}=-1,
\end{array}
$$


and moreover $K=\operatorname{Ker}(\mu)$ consists of 2-division points. Consider decompositions

$$
x=x_{1}+x_{2}+x_{3}+x_{4} \quad \text { and } \quad x^{\prime}=x_{1}^{\prime}+x_{2}^{\prime}+x_{3}^{\prime}+x_{4}^{\prime}
$$

with $x_{i}, x_{i}^{\prime} \in A_{i}$.

LEMMA 5.1. (a) $f_{1}$ and $f_{2}$ generate a group $\simeq \boldsymbol{Z} / 2 \boldsymbol{Z} \times \boldsymbol{Z} / 2 \boldsymbol{Z}$ if and only if $x_{1}, x_{2}, x_{1}^{\prime}$, $x_{3}^{\prime}$ and $x_{4}-x_{4}^{\prime}$ are 2-division points.

(b) $\Gamma=\left\langle f_{1}, f_{2}\right\rangle$ acts fixed point freely on $A$ if and only if

(i) $x_{1}+x_{2} \notin A_{3}+A_{4}$,

(ii) $x_{1}+x_{3} \notin A_{2}+A_{4}$, and

(iii) $g_{1}(y)+x \notin A_{2}+A_{3}$.

Proof. Assertion (a) is obvious. For (b)(i) note that $f_{1}$ acts fixed point freely if and only if $x \notin \operatorname{Im}\left(1-g_{1}\right)=A_{3}+A_{4}$. (ii) and (iii) mean that $f_{2}$ and $f_{1} f_{2}$ act fixed point freely.

Define biholomorphic maps $\tilde{f}_{1}$ and $\tilde{f}_{2}$ on $A_{1} \times A_{2} \times A_{3} \times A_{4}$ by

$$
\begin{aligned}
& \tilde{f}_{1}=t_{\left(x_{1}, x_{2}, x_{3}, x_{4}\right)} \circ(1 \times 1 \times(-1) \times(-1)), \\
& \tilde{f}_{2}=t_{\left(x_{1}^{\prime}, x_{2}^{\prime}, x_{3}^{\prime}, x_{4}^{\prime}\right)} \circ(1 \times(-1) \times 1 \times(-1)) .
\end{aligned}
$$

For $i=1,2$ the following diagram commutes

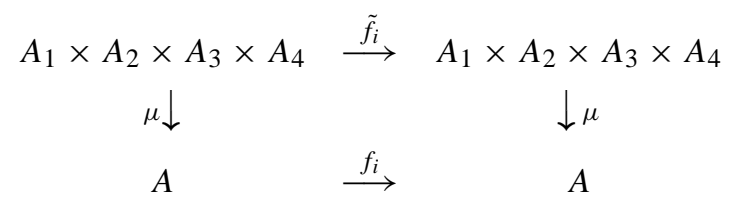

Using Lemma 5.1 (a) one easily checks that $\tilde{f}_{1}$ and $\tilde{f}_{2}$ generate a group isomorphic to $\boldsymbol{Z} / 2 \boldsymbol{Z} \oplus$ $\boldsymbol{Z} / 2 \boldsymbol{Z}$. Moreover, if $T$ denotes the group of translations of $A_{1} \times \cdots \times A_{4}$ by elements of $\operatorname{Ker}(\mu)$, then the sum $\left\langle f_{1}\right\rangle+\left\langle f_{2}\right\rangle+T$ is direct and

$$
\tilde{\Gamma}=\left\langle\tilde{f}_{1}\right\rangle \oplus\left\langle\tilde{f}_{2}\right\rangle \oplus T
$$

acts fixed point freely on $A_{1} \times \cdots \times A_{4}$. Hence we have

LeMmA 5.2. $X \simeq A_{1} \times A_{2} \times A_{3} \times A_{4} / \tilde{\Gamma}$.

LEMMA 5.3. Choosing suitable zero points of $A_{2}, A_{3}$ and $A_{4}$, we may assume that

$$
\tilde{f}_{1}=t_{\left(x_{1}, x_{2}, 0,0\right)} \circ g_{1} \text { and } \tilde{f}_{2}=t_{\left(x_{1}^{\prime}, 0, x_{3}^{\prime}, x_{4}^{\prime}\right)} \circ g_{2}
$$

with 2-division points $x_{1}, x_{2}, x_{1}^{\prime}, x_{3}^{\prime}, x_{4}^{\prime}, g_{1}=1 \times 1 \times(-1) \times(-1)$ and $g_{2}=1 \times(-1) \times$ $1 \times(-1)$.

PRoof. Let $z_{3} \in A_{3}$ with $2 z_{3}=x_{3}$. Then

$$
\tilde{f}_{1}\left(z_{3}\right)=-z_{3}+x_{3}=z_{3} .
$$


Choose $z_{3}$ as the new zero point of $A_{3}$ and let $\tilde{g}_{i}=t_{z_{3}} \circ g_{i} \circ t_{-z_{3}}$ for $i=1,2$ the corresponding automorphisms on $A_{3}$. Then for all $a_{3} \in A_{3}$ :

$$
\begin{aligned}
\tilde{g}_{1}\left(a_{3}\right) & =-a_{3}+2 z_{3}=\tilde{f}_{1}\left(a_{3}\right), \quad \text { and } \\
t_{x_{3}^{\prime}} \circ \tilde{g}_{2}\left(a_{3}\right) & =a_{3}+x_{3}^{\prime}=\tilde{f}_{2}\left(a_{3}\right) .
\end{aligned}
$$

Hence we may assume that $x_{3}=0$. The proof that we may assume $x_{2}^{\prime}=0$ is the same. Finally, let $z_{4} \in A_{4}$ with $2 z_{4}=x_{4}$. Then

$$
\tilde{f}_{1}\left(z_{4}\right)=-z_{4}+x_{4}=z_{4} .
$$

Choose $z_{4}$ as the new zero point of $A_{4}$ and let $\tilde{g}_{i}=t_{z_{4}} \circ g_{i} \circ t_{-z_{4}}$ for $i=1,2$ the corresponding automorphism on $A_{4}$. Then for all $a_{4} \in A_{4}$ :

$$
\tilde{g}_{1}\left(a_{4}\right)=g_{1}\left(a_{4}\right)-g_{1}\left(z_{4}\right)+z_{4}=-a_{4}+2 z_{4}=\tilde{f}_{1}\left(a_{4}\right) .
$$

According to Lemma 5.1 (a), $x_{4}^{\prime}=x_{4}+p_{4}$ with a 2-division point $p_{4}$ of $A_{4}$. Thus

$$
t_{p_{4}} \circ \tilde{g}_{2}\left(a_{4}\right)=g_{2}\left(a_{4}\right)-g_{2}\left(z_{4}\right)+z_{4}+p_{4}=-a_{4}+x_{4}^{\prime}=\tilde{f}_{2}\left(a_{4}\right) .
$$

Hence we may assume that $x_{4}=0$ and $x_{4}^{\prime}=p_{4}$ is a 2-division point.

Lemma 5.4. $\operatorname{Ker}(\mu) \cap\left\langle\left(x_{1}, x_{2}, 0,0\right),\left(x_{1}^{\prime}, 0, x_{3}^{\prime}, x_{4}^{\prime}\right)\right\rangle=\{0\}$.

Proof. Otherwise $\tilde{\Gamma}$ contains one of the automorphisms $g_{1}, g_{2}$ or $g_{1} g_{2}$ and thus admits fixed points on $A_{1} \times \cdots \times A_{4}$.

Combining everything we have proved most of the following theorem, the remaining assertions being easy to check.

THEOREM 5.5. For a variety $X$ of dimension $n(\geq 3)$ the following statements are equivalent:

(i) $X$ is an abelian hyperelliptic variety of type $(2,2)$.

(ii) There are

- abelian varieties $A_{i}$ of dimension $n_{i} \geq 0$ for $i=1, \ldots, 4$ with $\sum_{i=1}^{4} n_{i}=n$,

- 2-division points $x=\left(x_{1}, x_{2}, 0,0\right) \neq 0$ and $x^{\prime}=\left(x_{1}^{\prime}, 0, x_{3}^{\prime}, x_{4}^{\prime}\right) \neq 0$ of $A_{1} \times \cdots \times$ $A_{4}$ with $\left(x_{1}+x_{1}^{\prime}, x_{4}^{\prime}\right) \neq(0,0)$

- A subgroup $\tau$ of 2-division points of $A_{1} \times \cdots \times A_{4}$ with $\tau \cap\left\langle x, x^{\prime}\right\rangle=\{0\}$,

such that if $f_{1}=t_{x} \circ(1 \times 1 \times(-1) \times(-1))$ and $f_{2}=t_{x^{\prime}} \circ(1 \times(-1) \times 1 \times(-1))$ on $A_{1} \times \cdots \times A_{4}$ and $T$ denotes the group of translations by elements of $\tau$, then

$$
X \simeq A_{1} \times \cdots \times A_{4} / \Gamma
$$

with $\Gamma=\left\langle f_{1}\right\rangle \oplus\left\langle f_{2}\right\rangle \oplus T$.

Note that Theorem 5.5 can be easily applied to construct all abelian hyperelliptic varieties of type $(2,2)$.

6. Hyperelliptic threefolds. The first aim of this section is the proof of the following theorem. Finally, we complete the classification of all hyperelliptic threefolds.

THEOREM 6.1. Any hyperelliptic threefold is abelian. 
For the proof, assume that $X$ is a hyperelliptic threefold associated to a non abelian group. According to [UY] this group is necessarily the dihedral group $D_{4}$ of order 8 . Hence there is an abelian threefold $A$ and a group $\Gamma \subset \operatorname{Bihol}(A)$ acting fixed point freely on $A$ and isomorphic to $D_{4}$ such that $X=A / \Gamma$. Let $\Gamma$ be generated by $f_{1}$ and $f_{2}$ with $f_{1}^{4}=f_{2}^{2}=$ 1, $f_{2} f_{1} f_{2}=f_{1}^{-1}$. Then

$$
f_{1}=t_{x} \circ g_{1} \quad \text { and } \quad f_{2}=t_{y} \circ g_{2}
$$

with $g_{1}, g_{2} \in \operatorname{Aut}(A)$ with $g_{1}^{4}=g_{2}^{2}=1, g_{2} g_{1} g_{2}=g_{1}^{-1}$ and $x, y \in A$. Comparing the relations for $f_{1}, f_{2}$ with the relations for $g_{1}, g_{2}$, we obtain

LEMMA 6.2. (a) $g_{1}^{3}(x)+g_{1}^{2}(x)+g_{1}(x)+x=0$.

(b) $g_{2}(y)+y=0$.

(c) $\left(1+g_{1} g_{2}\right)(x)=-\left(g_{1}+g_{2}\right)(y)$.

Lemma 6.3. Let $B_{1}:=\operatorname{Ker}\left(1-g_{1}\right)^{0}$ and $B_{2}:=\operatorname{Im}\left(1-g_{1}\right)$. Then $\operatorname{dim} B_{1}=$ $1, \operatorname{dim} B_{2}=2$ and the addition map $\mu: B_{1} \times B_{2} \rightarrow A$ is an isogeny. Moreover, the group $G=\left\langle g_{1}, g_{2}\right\rangle$ acts on $B_{1}$ and $B_{2}$.

Proof. The group $G$ acts on the tangent space $T_{0} A=C^{3}$. This representation of $G$ must contain the two-dimensional representation of $G$, since otherwise the action of $G$ on $A$ would be commutative. The eigenvalues of $g_{1}$ of the two-dimensional representation are $\pm i$. Hence the one-dimensional representation has to be trivial on $g_{1}$. So $B_{1}$ is one-dimensional and $B_{2}$ is two-dimensional. Certainly, also $g_{2}$ acts on $B_{1}$ and $B_{2}$.

$B_{2}$ is an abelian surface with automorphism group $D_{4}$. These surfaces have been classified by Fujiki. In fact, according to Table 8 of $[\mathrm{F}]$, we know that $B_{2}$ is isomorphic to $E \times E$ with an arbitrary elliptic curve $E$ or the quotient of $E \times E$ by a group $H \simeq Z / 2 Z$ or $\simeq \boldsymbol{Z} / 2 \boldsymbol{Z} \times \boldsymbol{Z} / 2 \boldsymbol{Z}$ of diagonal 2-division points, and $D_{4}$ acts on $E \times E$ by

$$
g_{1}=\left(\begin{array}{cc}
0 & 1 \\
-1 & 0
\end{array}\right) \quad \text { and } \quad g_{2}=\left(\begin{array}{cc}
0 & 1 \\
1 & 0
\end{array}\right) \text {, }
$$

and on the quotients by the corresponding quotient actions. In any case, we write the elements of $B_{2}$ as pairs $\left(b, b^{\prime}\right), b, b^{\prime} \in E$. In the case of the quotients $E \times E / H$ we have to identify pairs which differ by a diagonal 2-division point of $H$. According to Lemma 3.3 (e),

$$
B_{1} \cap B_{2} \subseteq \Delta(2) \simeq Z / 2 Z \times Z / 2 Z
$$

where $\Delta$ denotes the image of the diagonal of $E \times E$ in $E \times E / H$. Hence we may write the elements $b$ of $A \simeq B_{1} \times B_{2} / B_{1} \cap B_{2}$ as

$$
b=b_{1}+\left(b_{2}, b_{2}^{\prime}\right)
$$

with $b_{1} \in B_{1}$ and $\left(b_{2}, b_{2}^{\prime}\right) \in B_{2}$. Again two such representations of $b \in A$ differ at most by 2-division points. 
Only two of the four one-dimensional representations of $D_{4}$ can occur since $g_{1} \mid B_{1}$ has to be the identity. Hence there are two cases

$$
\begin{array}{lll}
\text { Case 1: } & g_{1} \mid B_{1}=1, & g_{2} \mid B_{1}=1 . \\
\text { Case 2: } & g_{1} \mid B_{1}=1, & g_{2} \mid B_{1}=-1 .
\end{array}
$$

In both cases choose decompositions

$$
x=x_{1}+\left(x_{2}, x_{2}^{\prime}\right) \quad \text { and } \quad y=y_{1}+\left(y_{2}, y_{2}^{\prime}\right)
$$

for the translation points $x$ and $y$ of $f_{1}$ and $f_{2}$. Then the proof of Theorem 6.1 is completed if we show that in both cases the action of $\Gamma$ on $A$ is not fixed point free.

Case 1: According to Lemma 6.2, $g_{2}(y)=-y$. Hence $y$ is contained in the eigenspace of -1 of $g_{2}$, which is the antidiagonal $\widetilde{\Delta}:=\left\{(b,-b) \in B_{2}\right\}$ of $E \times E$ or its image $E \times E / H$. Hence we may assume $y=\left(y_{2},-y_{2}\right)$. On the other hand, $\operatorname{Im}\left(1-g_{2}\right)$ is just the antidiagonal $\widetilde{\Delta}$ of $B_{2}$, i.e., $y \in \operatorname{Im}\left(1-g_{2}\right)$. According to Lemma 3.1 this implies the assertion.

Case 2: Again we have $g_{2}(y)=-y$, which in this case is equivalent to $y_{2}^{\prime}=-y_{2}$. Hence $y=y_{1}+\left(y_{2},-y_{2}\right) \in B_{1}+\widetilde{\Delta}$. Now $\operatorname{Im}\left(1-g_{2}\right)=B_{1}+\widetilde{\Delta}$, i.e., $y \in \operatorname{Im}\left(1-g_{2}\right)$. Again Lemma 3.1 implies the assertion.

Hence we may assume that $X$ is a hyperelliptic threefold associated to an abelian group $G$ acting on an abelian threefold $A$. If $G$ is cyclic, then $X$ is necessarily of the type already described in Section 3. Hence we may assume that $G$ is not cyclic. Under these assumptions we have

LEMMA 6.4. The group $G$ is generated by two elements.

Proof. Suppose $G$ is non cyclic and cannot be generated by two elements. Then $G$ admits a subgroup isomorphic to $(\boldsymbol{Z} / p \boldsymbol{Z})^{3}$ with a prime $p$. Its generators, $g_{1}, g_{2}$ and $g_{3}$ say, cannot have a common eigenspace of the eigenvalue 1 , since there is no such automorphism group of an abelian surface (see [F]). But then it is easy to see that a suitable product of $g_{1}, g_{2}$ and $g_{3}$ does not admit an eigenvalue 1 .

Hence we may assume that $G \simeq \boldsymbol{Z} / d_{1} \boldsymbol{Z} \times \boldsymbol{Z} / d_{2} \boldsymbol{Z}$ with $d_{1} \mid d_{2}$ and is generated by $g_{i}$ of order $d_{i}$ for $i=1,2$. Then we have

LEMMA 6.5. There are two possibilities: Either

(i) The eigenspaces of 1 of $g_{1}$ and $g_{2}$ have a nontrivial intersection, or

(ii) $G \simeq Z / 2 Z \times Z / 2 Z$.

Proof. Suppose the contrary, i.e., $G$ is not of type (i) or (ii). We may choose the coordinates of $\boldsymbol{C}^{3}$ in such a way that $g_{1}=\operatorname{diag}\left(1, \alpha_{2}, \alpha_{3}\right)$ and $g_{2}=\left(\beta_{1}, \beta_{2}, 1\right)$ with $\alpha_{3} \neq$ $1 \neq \beta_{1}$. The element $g_{1} g_{2}$ has to admit an eigenvalue 1 , implying $\alpha_{2}=\beta_{2}^{-1}$. Hence $g_{1} g_{2}^{2}=$ $\operatorname{diag}\left(\beta_{1}^{2}, \beta_{2}, \alpha_{3}\right)$. Since $\beta_{2} \neq 1$ (otherwise $\alpha_{2}=\beta_{2}=1$ ), this implies $\beta_{1}=-1$. But then $g_{1}^{2} g_{2}=\operatorname{diag}\left(-1, \beta_{2}^{-1}, \alpha_{3}^{2}\right)$ gives $\alpha_{3}=-1$. Thus we have $g_{1}=\operatorname{diag}\left(1, \beta_{2}^{-1},-1\right), g_{2}=\operatorname{diag}$ $\left(-1, \beta_{2}, 1\right)$ with $\beta_{2} \neq \pm 1$. But now $g_{1} g_{2}^{3}=\operatorname{diag}\left(-1, \beta_{2}^{2},-1\right)$ admits no eigenvalue 1 , a contradiction. 
Applying Theorem 5.5, it is easy to construct all hyperelliptic threefolds of type $(2,2)$. So we are left with the case that $X$ is of type $\left(d_{1}, d_{2}\right)$ with $d_{2}>2$ such that $g_{1}$ and $g_{2}$ admit a common eigenspace of 1 . Since an elliptic curve admits only cyclic automorphism groups, Theorem 4.5 gives us an elliptic curve $B_{1}$ and an abelian surface $B_{2}$ admitting commuting automorphisms $g_{1}$ and $g_{2}$ of order $d_{1}$ and $d_{2}$ with $\operatorname{Fix}\left(g_{1}\right) \cap \operatorname{Fix}\left(g_{2}\right)$ finite, and points $\left(x_{1}, x_{2}\right),\left(x_{1}^{\prime}, x_{2}^{\prime}\right) \in B_{1} \times B_{2}$ and a group of translation $T$ of $B_{1} \times B_{2}$ with some additional properties such that

$$
X=B_{1} \times B_{2} /\left\langle f_{1}\right\rangle \oplus\left\langle f_{2}\right\rangle \oplus T
$$

with $f_{1}=t_{\left(x_{1}, x_{2}\right)} \circ\left(1 \times g_{1}\right)$ and $f_{2}=t_{\left(x_{1}^{\prime}, x_{2}^{\prime}\right)} \circ\left(1 \times g_{2}\right)$. Table 3 below gives all quadruples

TABLE 3.

\begin{tabular}{|c|c|c|c|c|}
\hline$\left(d_{1}, d_{2}\right)$ & $B_{2}$ & $g_{1}$ & $g_{2}$ & $\operatorname{Fix}\left(g_{1}\right) \cap \operatorname{Fix}\left(g_{2}\right)$ \\
\hline$(2,4)$ & $E \times E_{i}$ & $\left(\begin{array}{cc}-1 & 0 \\
0 & 1\end{array}\right)$ & $\left(\begin{array}{ll}1 & 0 \\
0 & i\end{array}\right)$ & $E(2) \times \operatorname{Fix}(i)$ \\
\hline$(2,4)$ & $E_{i} \times E_{i}$ & $\left(\begin{array}{cc}1 & 0 \\
0 & -1\end{array}\right)$ & $\left(\begin{array}{ll}i & 0 \\
0 & i\end{array}\right)$ & $\operatorname{Fix}(i) \times \operatorname{Fix}(i)$ \\
\hline$(2,4)$ & $E_{i} \times E_{i}$ & $\left(\begin{array}{cc}1 & 0 \\
1+i & -1\end{array}\right)$ & $\left(\begin{array}{ll}i & 0 \\
0 & i\end{array}\right)$ & $\operatorname{Fix}(i) \times \operatorname{Fix}(i)$ \\
\hline$(2,4)$ & $E_{i} \times E_{i}$ & $\left(\begin{array}{cc}1 & 0 \\
1 & -1\end{array}\right)$ & $\left(\begin{array}{ll}i & 0 \\
0 & i\end{array}\right)$ & $\{0\} \times \operatorname{Fix}(i)$ \\
\hline$(2,6)$ & $E \times E_{\rho}$ & $\left(\begin{array}{cc}-1 & 0 \\
0 & 1\end{array}\right)$ & $\left(\begin{array}{cc}1 & 0 \\
0 & -\rho\end{array}\right)$ & $E(2) \times\{0\}$ \\
\hline$(2,6)$ & $E_{\rho} \times E_{\rho}$ & $\left(\begin{array}{cc}-1 & 0 \\
0 & 1\end{array}\right)$ & $\left(\begin{array}{cc}-\rho & 0 \\
0 & -\rho\end{array}\right)$ & $\{0\}$ \\
\hline$(2,6)$ & $E_{\rho} \times E_{\rho}$ & $\left(\begin{array}{cc}1 & 0 \\
1 & -1\end{array}\right)$ & $\left(\begin{array}{cc}-\rho & 0 \\
0 & -\rho\end{array}\right)$ & $\{0\}$ \\
\hline$(2,6)$ & $E_{\rho} \times E_{\rho}$ & $\left(\begin{array}{cc}-1 & 0 \\
0 & 1\end{array}\right)$ & $\left(\begin{array}{cc}-\rho & 0 \\
0 & \rho^{2}\end{array}\right)$ & $\{0\} \times \operatorname{Fix}(\rho)$ \\
\hline$(2,12)$ & $E_{i} \times E_{\rho}$ & $\left(\begin{array}{cc}-1 & 0 \\
0 & -1\end{array}\right)$ & $\left(\begin{array}{cc}i & 0 \\
0 & -\rho\end{array}\right)$ & $\operatorname{Fix}(i) \times\{0\}$ \\
\hline$(3,3)$ & $E_{\rho} \times E_{\rho}$ & $\left(\begin{array}{cc}\rho & 0 \\
0 & \rho^{2}\end{array}\right)$ & $\left(\begin{array}{ll}\rho & 0 \\
0 & \rho\end{array}\right)$ & $\operatorname{Fix}(\rho) \times \operatorname{Fix}(\rho)$ \\
\hline$(3,3)$ & $E_{\rho} \times E_{\rho}$ & $\left(\begin{array}{cc}0 & 1 \\
-1 & -1\end{array}\right)$ & $\left(\begin{array}{ll}\rho & 0 \\
0 & \rho\end{array}\right)$ & $\operatorname{Fix}(\rho \mid \Delta)$ \\
\hline$(3,6)$ & $E_{\rho} \times E_{\rho}$ & $\left(\begin{array}{cc}\rho & 0 \\
0 & \rho^{2}\end{array}\right)$ & $\left(\begin{array}{cc}-\rho & 0 \\
0 & -\rho\end{array}\right)$ & $\{0\}$ \\
\hline$(3,6)$ & $E_{\rho} \times E_{\rho}$ & $\left(\begin{array}{cc}0 & 1 \\
-1 & -1\end{array}\right)$ & $\left(\begin{array}{cc}-\rho & 0 \\
0 & -\rho\end{array}\right)$ & $\{0\}$ \\
\hline$(3,6)$ & $E_{\rho} \times E_{\rho}$ & $\left(\begin{array}{ll}\rho & 0 \\
0 & 1\end{array}\right)$ & $\left(\begin{array}{cc}1 & 0 \\
0 & -\rho\end{array}\right)$ & $\operatorname{Fix}(\rho) \times\{0\}$ \\
\hline$(4,4)$ & $E_{i} \times E_{i}$ & $\left(\begin{array}{ll}i & 0 \\
0 & 1\end{array}\right)$ & $\left(\begin{array}{ll}1 & 0 \\
0 & i\end{array}\right)$ & $\operatorname{Fix}(i) \times \operatorname{Fix}(i)$ \\
\hline$(4,4)$ & $E_{i} \times E_{i}$ & $\left(\begin{array}{ll}i & 0 \\
i & 1\end{array}\right)$ & $\left(\begin{array}{cc}1 & 0 \\
-i & i\end{array}\right)$ & $\{0\} \times \operatorname{Fix}(i)$ \\
\hline$(6,6)$ & $E_{\rho} \times E_{\rho}$ & $\left(\begin{array}{rr}-\rho & 0 \\
0 & 1\end{array}\right)$ & $\left(\begin{array}{cc}1 & 0 \\
0 & -\rho\end{array}\right)$ & $\{0\}$ \\
\hline
\end{tabular}


$\left(B_{2}, g_{1}, g_{2}\right.$, Fix $\left(g_{1}\right) \cap$ Fix $\left.\left(g_{2}\right)\right)$ with $d_{2}>2$ which yield hyperelliptic threefolds in this way.

For the proof Fujiki's paper $[\mathrm{F}]$ is heavily used. According to $[\mathrm{F}]$, there are also some other abelian surfaces admitting a group of automorphisms of type $\left(d_{1}, d_{2}\right)$, but these are quotients of $B_{2}$ of table 3. Hence in order to construct the corresponding hyperelliptic threefold we may start with $B_{2}$ out of table 3. Applying Corollary 4.6, it is now easy to construct many hyperelliptic threefolds for any of the triples $\left(B_{2}, g_{1}, g_{2}\right)$ of the table: Choose a pair of $\left(d_{1}, d_{2}\right)$-division points $\left(x_{1}, x_{2}\right)$ of an elliptic curve $B_{1}$, a subgroup $T$ of $B_{1}$ and an embedding $\iota: T \hookrightarrow$ Fix $\left(g_{1}\right) \cap$ Fix $\left(g_{2}\right)$ such that $\Gamma=\left\langle x_{1}\right\rangle+\left\langle x_{2}\right\rangle+T$ is a direct sum. If $\Gamma$ acts by $\left(x_{1},\left(b_{1}, b_{2}\right)\right) \mapsto\left(t_{x_{1}}\left(b_{1}\right), g_{1}\left(b_{2}\right)\right),\left(x_{2},\left(b_{1}, b_{2}\right)\right) \mapsto\left(t_{x_{2}}\left(b_{1}\right), g_{2}\left(b_{2}\right)\right)$ and $\left(x,\left(b_{1}, b_{2}\right)\right) \rightarrow\left(t_{x}\left(b_{1}\right), t_{l}(x)\left(b_{2}\right)\right)$ for any $x \in T$, then $X=B_{1} \times B_{2} / \Gamma$ is a hyperelliptic threefold. One can apply Theorem 4.5 in order to construct all hyperelliptic threefolds of this type. This is a bit more complicated, but can be done separately in every case. However, there are too many cases, so this will be omitted.

\section{REFERENCES}

[BdF] G. BAGnera And M. DE Franchis, Sopra le superficie algebrique de hanno le coordinate del punto generico esprimibili con funzioni meromorfe quadruplamente periodiche di due parametri, Rend. della Reale Accad. dei Linci, Ser. V, XVI (1907), 492-498.

[BL] C. Birkenhake And H. Lange, Complex Tori, Progr. Math. 177, Birkhäuser Inc., Boston, MA, 1999.

[BGL] C. Birkenhake, V. Gonzalez and H. Lange, Automorphisms of 3-dimensional abelian varieties, Complex geometry of groups (Olmué, 1998), 25-47, Contemp. Math. 240, Amer. Math. Soc., Providence, RI, 1999.

[BPV] W. Barth, C. Peters and A. Van der Ven, Compact Complex Surfaces, Ergeb. Math. Grenzgeb. (3) 4, Springer-Verlag, Berlin, New York, 1984.

[ES] F. EnRIQUes And F. SEveri, Mémoire sur les Surfaces Hyperelliptiques, Acta Math. 32 (1909), $238-392$.

[F] A. FuJiki, Finite automorphism groups of complex tori of dimension two, Publ. Res. Inst. Math. Sci., 24 (1988), 1-97.

[GH] P. GRIFFITHS AND J. HARRIS, Principles of Algebraic Geometry, Pure and Applied Mathematics, John Wiley \& Sons, New York, 1978.

[J] F. E. A. Johnson, Flat algebraic manifolds, Geometry of low-dimensional manifolds, 1 (Durham, 1989), 73-91, London Math. Soc. Lecture Note Ser. 150, Cambridge Univ. Press, Cambridge, 1990.

[UY] K. UChIDA AND H. Yoshihara, Discontinuous groups of affine transformations of $\boldsymbol{C}^{3}$, Tôhoku Math. J. 28 (1976), 89-94.

MATHEMATISCHES INSITUT

DER UNIVERSITÄT ERLANGEN-NÜRNBERG

BISMARCKSTR. 1 1/2

91054 ERLANGEN

GERMANY 